PERSPECTIVES IN OPERATOR THEORY

BANACH CENTER PUBLICATIONS, VOLUME 75

INSTITUTE OF MATHEMATICS

POLISH ACADEMY OF SCIENCES

WARSZAWA 2007

\title{
SUBNORMALITY FROM BOUNDED VECTORS
}

\author{
FRANCISZEK HUGON SZAFRANIEC \\ Institute of Mathematics, Jagiellonian University \\ Reymont St. 4, 30-059 Kraków, Poland \\ E-mail: Franciszek.Szafraniec@uj.edu.pl
}

For a densely defined operator $S$ in a Hilbert space $\mathcal{H}$ having invariant domain, that is, $S \mathcal{D}(S) \subset \mathcal{D}(S)$, consider the following positive definiteness condition:

$$
\sum_{i, j=0}^{p}\left\langle S^{i} f_{j}, S^{j} f_{i}\right\rangle \geq 0, \quad f_{0}, \ldots, f_{p} \in \mathcal{D}(S) .
$$

Moreover, for a densely defined operator $A$ in $\mathcal{H}, f \in \cap_{n=1}^{\infty} \mathcal{D}\left(A^{n}\right)$ is said to be a bounded vector if

$$
\left\|A^{n} f\right\| \leq a b^{n}, \quad n \in \mathbb{N}
$$

with $a$ and $b$ depending on $f$.

QUESTION. Is $S$ subnormal if it satisfies (1) and the set of bounded vectors of $S^{*}$ is dense?

\section{References}

[1] F. H. Szafraniec, Bounded vectors for subnormality via a group of unbounded operators, Contemp. Math. 341 (2004), 113-118. 